\title{
Vehicle Classification Based on the Radar Measurement of Height Profiles
}

\author{
Ildar Urazghildiiev, Rolf Ragnarsson, Pierre Ridderström, Anders Rydberg, Member, IEEE, Eric Öjefors, \\ Kjell Wallin, Per Enochsson, Magnus Ericson, and Göran Löfqvist
}

\begin{abstract}
The problem of classifying road vehicles according to vehicle type is considered. The proposed solution is based on using vehicle height and length and height profiles obtained by a microwave (MW) radar sensor. We show that if the radar sensor satisfies certain requirements, then a precise feature vector can be extracted, and simple deterministic algorithms can be applied to determine the vehicle class. Field trials using a spread-spectrum MW radar sensor system operating on these principles have been carried out. They confirm that accurate classification of a large number of vehicle classes can be reached.
\end{abstract}

Index Terms-Classification, feature extraction, height profile, road vehicles, spread-spectrum radar.

\section{INTRODUCTION}

$\mathbf{T}$ HE PROBLEM of the classification of road vehicles arises in many areas such as truck volume estimating, traffic planning, roadway tolling, etc.; see e.g., [1]-[4] and references therein. To solve this problem, a number of solutions have been proposed. The known methods of vehicle classification are based on using such kinds of sensors as pneumatic road tubes, inductive loops, magnetic sensors, piezoelectric sensors, video cameras, infrared lasers sensors, microwave (MW) radars, and ultrasonic sensors [3]-[7]. Any kind of sensor will provide a specific mechanism of classifying vehicles and has its own advantages and disadvantages. Since user needs and classification conditions can differ, no sensors and corresponding techniques have proven to be the best for all possible applications [2], [3]. Therefore, any new classification technique providing specific advantages can be of great interest for the highway agencies.

Manuscript received February 23, 2006; revised June 19, 2006, October 4 , 2006, October 23, 2006, October 30, 2006, and November 3, 2006. This work was supported in part by Sensys Traffic AB, Sweden, and the Carl Trygger Foundation, Sweden, under Grant CTS 02-257. The Associate Editor for this paper was R. W. Goudy.

I. Urazghildiiev is with the Laboratory of Ornithology, Cornell University, Ithaca, NY 14850 USA (e-mail: iru2@ cornell.edu).

R. Ragnarsson and P. Ridderström are with the Sensys Traffic AB, 75183 Uppsala, Sweden (e-mail: Rolf.Ragnarsson@sensystraffic.se; Pierre. Ridderstrom@sensystraffic.se).

A. Rydberg and E. Öjefors are with the Department of Signals and Systems, Uppsala University, 75105 Uppsala, Sweden (e-mail: Anders.Rydberg@ signal.uu.se; Eric.Ojefors@signal.uu.se).

K. Wallin, P. Enochsson, M. Ericson, and G. Löfqvist are with Sensys Traffic AB, 55002 Jönköping, Sweden (e-mail: Kjell.Wallin@ sensystraffic.se; Per.Enochsson@sensystraffic.se; Magnus.Ericson@sensystraffic.se; Goran. Lofqvist@ sensystraffic.se).

Digital Object Identifier 10.1109/TITS.2006.890071
In certain situations, some benefits can be provided by MW radar sensors. MW sensors do not require installation in the roadway, thus making sensor calibration and maintenance easier and less disruptive. This is important for use in high-volume urban freeways, highways, and other locations where access to the roadway is extremely limited and expensive. Another strength is that MW radar sensors are largely immune to adverse weather and light conditions or vibrations. Such properties have lead to intense practical interest in MW classification systems [2], [3].

In various traffic management applications, roadsidemounted and forward-looking frequency-modulated continuous wave (FMCW) and noise-correlation radar units combined with continuous-wave (CW) Doppler sensors are commonly used [3], [8], [9]. Both of these MW radar sensors are primarily intended for extraction of vehicle length and shape information. However, high resolution in the distance domain is required to obtain accurate vehicle shape information from a roadsidemounted sensor. As a result, the FMCW radar classification system considered in [8] gives only $75 \%$ accuracy when separating traffic into five categories.

A forward-scattering CW Doppler radar was used to obtain a vehicle signature in [10]. The vehicle signatures obtained using Doppler radar were also used in [11] and [12]. Because of the high variability in geometric shapes of vehicles, extracting sufficient information from the signatures for the detailed classification of vehicles is a difficult task. Therefore, such types of classification systems are feasible for categorization into a relatively small number of vehicle classes such as small, medium, and large cars [10], [11] or tracked and wheeled vehicles [12]. The reported classification accuracy for these systems is $79.7 \%$ [10], 89\% [11], and $83 \%$ [12].

To enhance classification accuracy and increase the number of vehicle types being classified, a vehicle classification system using down-looking spread-spectrum MW radar was proposed in [13]. In this system, the sensor was mounted above the roadway in such a way that vehicles pass directly below the sensor. Such installation made it possible to use the vehicle height profile as the main feature for classification. As a result, the reported classification accuracy was $99 \%$ for five vehicle types and a data set of 1706 vehicles. Another strength of using a down-looking sensor is that vehicles do not occlude each other from the sensor's point of view, which is important for urban areas.

The classification algorithm proposed in [13] is based on representing vehicles in a parameter space including the vehicle 
height and length and height profiles. Including the vector of height profile to the feature space is a sufficient condition for enhancing the distinguishability of vehicles having similar lengths and heights. However, for certain vehicle types, using the height profile is not necessary. Many vehicles, such as small passenger cars or big trucks, can be unambiguously represented in feature spaces of smaller dimension based on height and length. Investigations show that the portion of passenger cars and big trucks can be $90 \%$ and more [4]. Therefore, the classification algorithm proposed in [13] requires a great number of redundant measurements of vehicle height profiles. As a result, practical implementation of the algorithm [13] involves unnecessary labor to design patterns for many vehicle types and requires high computational costs when calculating the height profile estimates.

The main goal of this paper is to simplify the approach [13] based on down-looking spread-spectrum MW radar. We consider a standard problem of classification of vehicles according their type. To solve this problem, we develop a new classification technique. The basic idea behind the proposed technique is to use different feature spaces for different vehicle types based on their linear dimensions and shape. We show that using feature spaces of lower dimension does not affect the classification performance while simplifying the classification algorithm. We also formulate basic requirements for the MW sensor and demonstrate the performance of the proposed technique in a real traffic situation. Tests for 2945 vehicles separated by the classes of car, car with trailer, van, bus, single-unit truck (SUT), and multitrailer truck (MTT) were conducted.

This paper is organized as follows: In Section II, we make the basic assumption, introduce the data model, and formulate the problem. The proposed vehicle classification technique is considered in Section III. The basic sensor requirements are formulated in Section IV. A vehicle classification system using a down-looking spread-spectrum MW radar sensor is presented in Section V. The test results demonstrating the system performance are also given in this section. The conclusions are presented in Section VI. In this paper, the following notations are used:

\begin{tabular}{|c|c|}
\hline$M$ & Number of classes. \\
\hline$C_{1}, \ldots, C_{M}$ & Classes of vehicles. \\
\hline$K_{m}$ & $\begin{array}{l}\text { Number of vehicle types comprising } \\
\text { the } m \text { th class. }\end{array}$ \\
\hline$K$ & $\begin{array}{l}\text { Number of different vehicle types to } \\
\text { be classified. }\end{array}$ \\
\hline$N$ & Number of data samples per vehicle. \\
\hline $\boldsymbol{h}=[h(1), \ldots, h(L)]^{T}$ & $\begin{array}{l}L \text {-dimensional vector of height } \\
\text { profile. }\end{array}$ \\
\hline$h$ & Maximum vehicle height. \\
\hline$l$ & Maximum vehicle length. \\
\hline$F^{P}$ & $\begin{array}{l}\text { Linear metrical } P \text {-dimension vector } \\
\text { space. }\end{array}$ \\
\hline $\boldsymbol{p}_{k}^{(m)} \in F^{P}$ & $\begin{array}{l}\text { Pattern vector of the } k \text { th vehicle type } \\
\text { belonging to the } m \text { th class. }\end{array}$ \\
\hline $\boldsymbol{f} \in F^{P}$ & Feature vector. \\
\hline$d\left(\boldsymbol{f}_{i}, \boldsymbol{f}_{j}\right)$ & $\begin{array}{l}\text { Scalar distance between two vectors } \\
\boldsymbol{f}_{i} \text { and } \boldsymbol{f}_{j} \in F^{P} \text {. }\end{array}$ \\
\hline
\end{tabular}

\section{Data Model and Problem Formulation}

Every vehicle can be represented as a vector in some feature space. The features commonly used in practice include the number of axles and the distance between them. For instance, the axle-based classification scheme is used by the U.S. Federal Highway Administration. This scheme comprises 13 categories such as motorcycles, passenger cars, other two-axle singleunit vehicles, buses, and nine types of trucks [2]. The classification scheme used in the toll plazas in New York City is also based on axle parameters and comprises ten vehicle classes, such as motorcycles, cars and vans, five classes of trucks with two to six axles, cars towing trailers, and twoaxle and three-axle buses [4]. The British M6 Toll uses a sixcategory classification scheme with similar classes [14], which are also defined in terms of vehicle axles. One can see that many of the aforementioned vehicle types such as motorcycles, passenger cars, light trucks, buses, heavy trucks, and others have distinguishable geometric shapes. Therefore, these types can be specified in terms of height profiles. This fact is used to develop a classification technique considered here.

We make the following basic assumptions.

A1) The classification scheme comprises $M$ classes $C_{1}, \ldots, C_{M}$. Each class $C_{m}$ consists of $K_{m}>0$ vehicle types so that $\Sigma_{m=1}^{M}\left(K_{m}\right)=K$. For instance, the class "passenger car" can include several types such as "sedan" or "SUV."

A2) Each type of the vehicle is completely specified by its unique geometric shape. The geometric shape of the $k$ th vehicle type is described by the $L$-dimensional vector of height profile $\boldsymbol{h}_{k}, k=1, \ldots, K$.

A3) The height profiles of the vehicles are measured by the down-looking spread-spectrum MW radar mounted overhead. When the vehicle passes the roadway below the sensor, the data on the sensor output can be modeled as

$$
x(n)=h(n)+\varepsilon(n), \quad n=1, \ldots, N
$$

where $h(n)$ is the height of the reflecting plane on a vehicle surface, $\varepsilon(n)$ is the random value describing the measurement error, and $N$ is the number of samples per vehicle. Since passing vehicles have different speeds and lengths, the value of $N$ is assumed to be random. We also assume the speed of every vehicle passing the surveillance area to be constant.

The problem can be formulated as follows: Given the observations $x(n)$, assign the vehicle to one of the classes $C_{1}, \ldots, C_{M}$.

\section{Classification Technique}

The solution of the classification problem includes extracting the feature vector from the sensor output (1) and mapping it onto predefined classes. Since classes are defined as subspaces in some feature space, development of the feature space is a critical issue. 


\section{A. Feature Space}

We define a feature space as a linear metrical $P$-dimension vector space $F^{P}$. The structure of the feature space is restricted by A3). The basic vehicle parameters that can be extracted from samples $x(n)$ include height profile $\boldsymbol{h}$ and maximum height (or merely height) $h$. In many cases, the classification sensor is used along with the Doppler radar, making it possible to measure vehicle speed [3], [4], [11]. If the speed of the vehicle is available, then vehicle length $l$ can also be included in the feature space. Thus, using MW radar sensors, the full feature space with the following structure is available:

$$
F^{P}=\left\{\boldsymbol{f} \mid \boldsymbol{f}=\left[\boldsymbol{h}^{T}, \boldsymbol{b}^{T}\right]^{T}\right\}
$$

where $\boldsymbol{b}=[h, l]^{T}, P=L+2$, and the symbol $(\cdot)^{T}$ denotes transpose. In space $F^{P}$, the pattern vector of the $k$ th vehicle type being classified can be defined as

$$
\boldsymbol{p}_{k}=\left[\boldsymbol{h}_{k}^{T}, \boldsymbol{b}_{k}^{T}\right]^{T} \in F^{P}, \quad k=1, \ldots, K .
$$

From A2), it follows that any of the $m$ th class of vehicles can be defined as a set of patterns so that $C_{m}=$ $\left\{\boldsymbol{p}_{1}^{(m)}, \boldsymbol{p}_{2}^{(m)}, \ldots, \boldsymbol{p}_{K_{m}}^{(m)}\right\} \subset F^{P}$

The basic requirement to the feature space is the unambiguous representation of classes. The classification problem has a unique solution if sets $C_{1}, \ldots, C_{M}$ are nonintersecting so that

$$
C_{m} \bigcap C_{n}=\varnothing
$$

for all $m \neq n$. By now, variability of vehicles is high enough so that the satisfaction of condition (4) cannot be guaranteed for any classification scheme. For instance, condition (4) holds true for such vehicle types as motorcycles, passenger cars, buses, and heavy trucks. However, trucks with different numbers of axles but similar shapes cannot be distinguished in $F^{P}$. In practice, increasing the dimension of vector $\boldsymbol{h}$ can relax the ambiguity problem. Asymptotically, when $L$ tends to infinity, only vehicles with identical height profiles are nondistinguishable in space $F^{P}$. Thus, taking into account A1) and A2), including the vector of the height profile to the feature space, a sufficient condition for the classification scheme is unambiguous. However, employing vectors of height profiles requires considerable effort in creating the patterns and obtaining height profile estimates. Therefore, the question of whether this condition is also necessary is of great practical interest.

Let us introduce feature space $F^{2}=\{\boldsymbol{b}\}$, consisting of vehicle heights and lengths. In this space, the ambiguity level is high enough, even when relatively simple classification schemes are introduced. For instance, as it was reported in [2], the definition of such vehicle types as passenger vehicles, SUTs, combination trucks, and MTTs in the height-length space $F^{2}$ results in the unambiguous classification of $79.1 \%-81.9 \%$ of the vehicles. The height and length of 312 vehicle types as published by manufacturers are shown in Fig. 1. In this figure, vehicle types were categorized as passenger car, light truck, SUV-minivan, van, bus, and heavy SUT. In spite of the fact that many vehicle

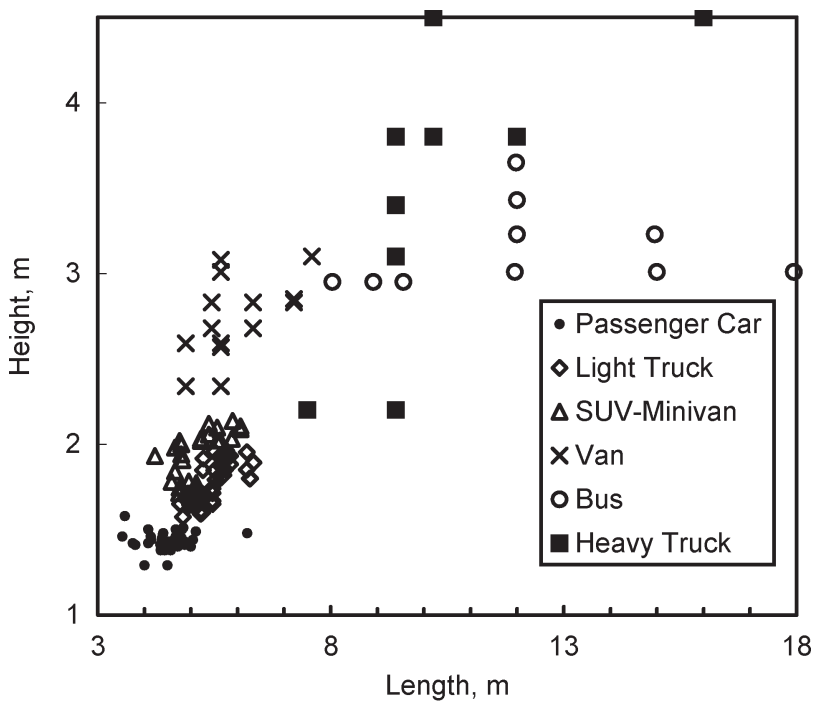

Fig. 1. Height and length of 312 vehicle types, as published by manufacturers. Vehicle types are categorized as classes passenger car, light truck, SUV-minivan, van, bus, and heavy truck.

types are intersecting in $F^{2}$, one can define the nonintersecting subsets $C_{m}^{*} \subseteq C_{m} \subset F^{2}$ such that

$$
C_{m}^{*} \bigcap C_{n}^{*}=\varnothing
$$

for all $m \neq n$. In particular, the heights and lengths of a great number of passenger cars are less than the corresponding dimensions of trucks, buses, and other big vehicles. Then, the nonintersecting subclasses $C_{m}^{*}$ in space $F^{2}$ can be defined as the following continuous sets:

$$
C_{m}^{*}=\left\{\boldsymbol{b} \mid h_{\min }^{(m)}<h \leq h_{\max }^{(m)}, l_{\min }^{(m)}<l \leq l_{\max }^{(m)}\right\}
$$

where $h_{\min }^{(m)}, h_{\max }^{(m)}, l_{\min }^{(m)}$, and $l_{\max }^{(m)}$ are the minimum and maximum heights and lengths representing the height and length boundaries of subclass $C_{m}^{*}$, respectively. Height and length classification boundaries specifying nonintersecting subclasses for classes car, car with trailer, van, bus, SUT, and MTT are represented in Section V.

Without loss of generality, many low-dimension feature spaces similar to $F^{2}$, such as $F^{1}=\{h\}$ and $F^{1}=\{l\}$, can be introduced based on the properties of the available sensors. In these spaces, nonoverlapping subspaces (5) and (6) unambiguously representing a certain percentage of the vehicle types can be defined.

Thus, for many vehicle types, using the full feature space (2) is not necessary. A classification technique based on using a combination of low-dimension and high-dimension feature spaces is considered in Section III-C.

\section{B. Feature Vector Extraction}

Feature vector extraction involves the single-valued transformation of samples $x(n)$ into feature vector $\boldsymbol{f}$. The $N$ samples of height measurements (1) can be divided into $L$ (possibly overlapping) segments with $W \geq[N / L]$ samples in each segment, where symbol $[c]$ denotes the integer part of $c$. For any $j$ th 
segment, some estimate of the vehicle height can be calculated. The choice of an acceptable estimation algorithm depends on the statistical properties of the measurement errors $\varepsilon(n)$. The results of our experiments show that there is a nonzero probability of outliers in samples $x(n)$. In the presence of outliers, using mean values results in degeneration of estimation accuracy. Therefore, we propose to calculate the $j$ th estimate of height as a median value of the corresponding samples $x(n)$

$$
\hat{h}(j)=\operatorname{med}\left\{x\left(n_{\min }\right), \ldots, x\left(n_{\max }\right)\right\}, \quad j=1, \ldots, L
$$

where $n_{\min }$ and $n_{\max }$ are the minimum and maximum indexes of samples $x(n)$ that fall into the $j$ th segment, respectively. The vector of height profile estimates can be represented as $\hat{\boldsymbol{h}}=$ $[\hat{h}(1), \ldots, \hat{h}(L)]^{T}$.

The estimates of parameters $h$ and $l$ can be obtained in different ways. For instance, a trivial vehicle height estimate can be calculated as $\hat{h}=\max \{\hat{\boldsymbol{h}}\}$. However, due to outliers, this estimate may have a large error. Therefore, we propose the robust technique of vehicle height estimation.

Denote the normalized histogram of vehicle height estimates as $F\left(g_{i}\right), i=1,2, \ldots$ For a given height profile $\boldsymbol{h}$, the function $F\left(g_{i}\right)$ is defined as the percentage of height samples (1) that fall within the height interval $\left[g_{i}-\Delta / 2, g_{i}+\Delta / 2\right]$, where $\Delta=$ $g_{i+1}-g_{i}$. If a vehicle has a flat surface that is parallel to the ground, the histogram $F\left(g_{i}\right)$ has a peak at point $\tilde{g}$, which is close to the height of such surface. The magnitude of the peak is determined as

$$
F(\tilde{g}) \approx \frac{l_{0}}{l}
$$

where $l_{0}$ is the length of the surface. In a general case, vehicles might have $q \geq 1$ flat surfaces parallel to the ground. It results in that the corresponding height distribution function has $q$ local peaks at points $\tilde{g}_{1}, \ldots, \tilde{g}_{q}$. We propose to define the height of a vehicle as $h=\max \left\{\tilde{g}_{1}, \ldots, \tilde{g}_{q}\right\}$. Based on this definition, the maximum height of a vehicle can be estimated as

$$
\hat{h}=\arg \max _{i} F\left(\tilde{g}_{i}\right) .
$$

We also require that $F(\hat{h}) \geq C$, where $C$ is a threshold determined according to (8) in terms of the minimum length of the surface. For many vehicles, the height of the cabin or other similar surfaces can be used as a measure of the vehicle's height. Observe that a small number of outliers in the height estimates of the corresponding flat surface do not affect the estimation accuracy of the proposed algorithm. An example of the histogram of vehicle height estimates is considered in Section V-B.

The vehicle's length can be estimated by combining the vehicle velocity measurements from a Doppler radar sensor with the measurement of the vehicle height profile as

$$
\hat{l}=N T_{0} v
$$

where $T_{0}$ is the radar sampling period, and $v$ is the speed of the vehicle.
Thus, after the transformation (7) and finding the estimates of the vector $\hat{\boldsymbol{b}}=[\hat{h}, \hat{l}]^{T}$, we obtain the feature vector estimate that can be represented as

$$
\boldsymbol{f}=\left[\hat{\boldsymbol{h}}^{T}, \hat{\boldsymbol{b}}^{T}\right]^{T}=\boldsymbol{p}_{k}+\boldsymbol{\eta}
$$

where $\boldsymbol{p}_{k}$ is the pattern of the observed vehicle, and $\boldsymbol{\eta} \in F^{P}$ is a vector of estimation error.

\section{Classification}

Regarding vector $\boldsymbol{f}, M$ hypotheses can be introduced. We denote the hypotheses as $H_{1}, \ldots, H_{M}$. The $H_{m}$ hypothesis states that feature vector $f$ belongs to class $C_{m}$. The decision to accept one of the hypothesis $H_{m}$ can be made using many different strategies. Optimal classification algorithms such as Bayesian, maximum likelihood, min-max, and others [15], [16] can be developed using the statistical decision theory. The strength of the optimal algorithms is that they minimize the average number of classification errors. However, implementation of the optimal methods requires knowledge of the probability density function of the feature vector (11). In practice, statistical distribution of vector $\boldsymbol{\eta}$ is unknown, and development of the acceptable model for the entire set of vehicles of interest is not an easy task. To avoid this difficulty, we use the fact that the sensor is mounted in close vicinity of the vehicles. This enables us to assume that the mean square error of the feature vector estimate is negligibly small, as compared to the vehicle dimensions. We also assume that the patterns stored in the database cover all basic vehicle types so that for almost any feature vector, the following condition holds true:

$$
E\left\{d^{2}(\boldsymbol{f}, \boldsymbol{p})\right\}=E\left\{\|\boldsymbol{\eta}\|^{2}\right\} \ll D_{\min }^{2}
$$

where $D_{\min }=\min _{i \neq j} d\left(\boldsymbol{p}^{(i)}, \boldsymbol{p}^{(j)}\right), i, j=1, \ldots, M$ is the minimum interclass distance, and symbol $E\{\cdot\}$ denotes expectation. Thus, we can neglect the influence of the measurement error and use the deterministic approach to the problem of classification of vehicles.

The classification technique being proposed is based on using the feature spaces of low dimension along with full space $F^{P}$. As a low-dimension space, we use feature space $F^{2}$, although any other spaces similar to those considered in Section III-A can be used. The classification technique involves the following steps.

Step 1) For the given samples $x(n)(1)$, calculate the vector $\hat{\boldsymbol{b}}=[\hat{h}, \hat{l}]^{T} \in F^{2}$ using (9) and (10).

Step 2) For $m=1, \ldots, M$, check the conditions (6). If there is any $C_{m}^{*}$ such that $\hat{\boldsymbol{b}} \in C_{m}^{*}$, then accept $C_{m}$ as a vehicle class estimate and terminate the algorithm. Otherwise, go to Step 3).

Step 3) Calculate the vector of height profile $\hat{h}$ (7) and feature vector $f \in F^{P}$ (11).

Step 4) Compute distances $d\left(\boldsymbol{f}, \boldsymbol{p}_{k}^{(m)}\right), \quad k=1, \ldots, K_{m}$, $m=1, \ldots, M$. 
Step 5) Find the indexes.

$$
[\hat{k}, \hat{m}]=\arg \min _{k, m} d\left(\boldsymbol{f}, \boldsymbol{p}_{k}^{(m)}\right) .
$$

Step 6) Accept class $C_{\hat{m}}$ as the vehicle class estimate.

Observe that the algorithm considered in [13] involves Steps 1) and 3)-5) for each vehicle. In the proposed technique, a certain percentage of vehicles can be classified in Steps 1) and 2). Thus, for many vehicle types, creating height profile patterns and measuring height profiles can be avoided. This simplifies the practical implementation of the proposed technique.

One of the "degrees of freedom" in Step 4) is the metric $d(\boldsymbol{f}, \boldsymbol{p})$. The choice of a metric can be based on the observation that estimation accuracy varies for different vehicle parameters. In particular, the mean square error of the vehicle height estimate is usually smaller as compared to an arbitrary point on the height profile. Apart from this, the differences between vehicle types are larger over length than over height. Therefore, it is natural to use different weights for different vehicle parameters. Then, we arrive at the an anisotropic Euclidian metric, which is described by

$$
d(\boldsymbol{f}, \boldsymbol{p})=(\boldsymbol{f}-\boldsymbol{p})^{T} \boldsymbol{W}(\boldsymbol{f}-\boldsymbol{p})
$$

where $\boldsymbol{W} \in R^{P \times P}$ is a weighted matrix, which is a symmetric and positive definite. Since all the components of the feature space are determined independently, it can be diagonal, i.e., $\boldsymbol{W}=\operatorname{diag}\left\{w_{1}, \ldots, w_{P}\right\}$, where weight $w_{i}$ can be the inverse proportional to the mean square error of the $i$ th feature estimate.

\section{SENSOR REQUiREMENTS}

To apply the data model (1), the MW sensor must satisfy certain requirements. The basic requirements include the following.

R1) A plane radiation pattern that ensures wide road coverage transverse to traffic flow and narrow coverage along the traffic flow. This requirement is needed to guarantee that all the relevant scatterers on the vehicle surface are at approximately the same distances to the antenna, and they can be lumped together as one scatterer. At the same time, we want the sensor to cover the full width of a lane.

R2) High distance resolution, which enables us to resolve two scatterers in the antenna beam, such as vehicle hood and road surface or hood and roof. The utilization of transmitted signals with spread spectrum and wide bandwidth can satisfy this requirement.

R3) High sampling frequency $F_{0}$. This is needed to get $N>L$ samples for all vehicles. Let $l_{\min }$ be the minimum length of a vehicle to be classified and $v_{\max }$ be the maximum speed. Then, R3) means that $F_{0} \geq$ $L v_{\max } / l_{\min }$.

R4) A wide dynamic range in terms of return amplitudes. If the sensor dynamic range is less than the maximum values of fluctuations in the return signal, then large "anomalous" errors that prevent correct vehicle height

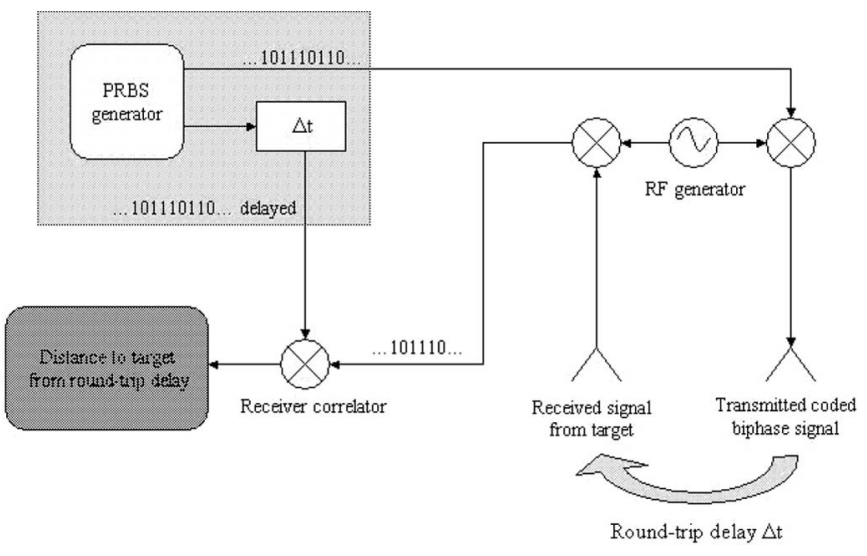

Fig. 2. Block diagram of the MW radar sensor.

estimation can arise. For instance, the returns of extremely low and extremely large amplitudes can be out of analog-to-digital (A/D) converter range. This results in a dramatically increased height estimation error.

In the next section, we demonstrate how well these requirements can be met in a real MW spread-spectrum sensor and which classification accuracy can be reached.

\section{Field Trials}

Joint development by Sensys Traffic AB and Uppsala University has resulted in a classification system that includes two radar sensors used for height profile and speed measurements, as well as a standard personal computer on which the signal processing algorithms were implemented. The first goal of the field trials was to confirm the possibility of developing a vehicle classification system based on the MW radar measurement of height profile. We demonstrate that it is possible to develop a MW sensor satisfying requirements R1)-R4). The second goal is to test the vehicle classification technique and estimate its efficiency in real traffic situations.

\section{A. Test-Bed System}

The spread-spectrum radar sensor, as shown in Fig. 2, consists of several parts: a pseudorandom binary sequence (PRBS) generator, an RF unit, antennas, and a correlator. The PRBS generator produces a sequence of binary states that is used to modulate the radio signal generated in the RF unit. A transmitted signal with pseudorandom phase modulation is used to ensure low dependence on the Doppler shift of the signal spectrum and cancellation of interference as well as to provide waveform flexibility. The resulting modulated signal is transmitted from a separate transmitter antenna directed down toward the road surface. The received signal is amplified before being split and demodulated to obtain the in-phase (I) and the quadrature $(\mathrm{Q})$ channels. In the correlator, the demodulated signals are compared to a time-delayed binary sequence from the PRBS generator to determine the round-trip time (transmitter-target-receiver) of the transmitted signal. From the round-trip time, the sensor-target distance is determined with a resolution of $0.244 \mathrm{~m}$ for a single measurement [c.f. R2)]. When 
no vehicle is present, an echo from the road surface is received, which provides a baseline for vehicle height measurements. The I and the $\mathrm{Q}$ channel outputs are digitized with a 16-bit $\mathrm{A} / \mathrm{D}$ converter [c.f. R4)].

The radar operates at a frequency of $24.15 \mathrm{GHz}$ with beam widths of $8^{\circ}$ (along the traffic flow) $\times 24^{\circ}$ (transverse to the traffic flow) and a transmitted power of less than $100 \mathrm{~mW}$ effective isotropic radiated power [c.f. R1)]. The frequency of height measurements is $1160 \mathrm{~Hz}$; thus, the height of a 5-m-long car traveling at $180 \mathrm{~km} / \mathrm{h}$ is sampled at 125 different locations along the length of the vehicle [c.f. R3)]. An MW Doppler sensor was used to estimate the speed and length of vehicles. As a result, the feature vector with the structure (2) was available in the tests.

It is worthwhile to note that the MW radar sensor has a relatively low cost and does not require high installation and maintenance costs when an appropriate bridge or gantry is available. Note also that the classification process makes moderate demands on computational capacity. The number of multiplications required in Step 4), which is subject to (14), is $O(K P)$. Real-time operation can thus be achieved with a relatively simple processor.

\section{B. Field Trial Results}

The test data were obtained from a field site on the E4 highway in Uppsala, Sweden, from November 2001 to June 2002. The highway has two lanes in both directions. The mean speed of the traffic flow was about $70 \mathrm{~km} / \mathrm{h}$. For traffic measurements, the radar was mounted $5.5 \mathrm{~m}$ vertically above the plane of the road surface. The pedestrian bridge served as the mounting point for the sensor hardware. The site was instrumented by a video ground truth camera.

The first stage of the field trials was focused on the determination of the root-mean-square error (RMSE) of the estimates of a vehicle's height profile $\hat{\boldsymbol{h}}$ and the corresponding maximum height $\hat{h}$ and length $\hat{l}$. Typical examples of height samples (1) given by the MW radar sensor as well as visual images of the corresponding vehicles are depicted in Figs. 3 and 4. For the truck represented in Fig. 4, the normalized histogram of the height estimates $F(g)$ is shown in Fig. 5. One can see from this figure that the histogram has $q=2$ peaks at points $\tilde{g}_{1}=3.4 \mathrm{~m}$ and $\tilde{g}_{2}=2.9 \mathrm{~m}$, corresponding to the heights of the box and the cabin, respectively. The threshold for the histogram was set up as $C=0.1$. Since $F\left(\tilde{g}_{1}\right)>F\left(\tilde{g}_{2}\right) \geq C$, the value $\tilde{g}_{1}=3.4 \mathrm{~m}$ was accepted as the height of the truck. Statistical analysis of the measurement results reveals that the RMSE does not exceed $0.1-0.15 \mathrm{~m}$ for the estimates $\hat{h}(j)$ of the elements of the vector of height profile (7) and $0.02-0.05 \mathrm{~m}$ for the estimate of the maximum height of the vehicle $\hat{h}(9)$.

The Doppler radar sensor gives the speed of the vehicles to be classified to within $0.13 \mathrm{~m} / \mathrm{s}$ in the range of speeds $v>5 \mathrm{~m} / \mathrm{s}$. For small vehicles (such as passenger cars), this yields an RMSE for length estimates $\hat{l}$ of not more than $0.15 \mathrm{~m}$ in the range of speeds $5-50 \mathrm{~m} / \mathrm{s}$. Thus, the first stage of field trials shows that the measurement errors are much smaller than the typical dimensions of the vehicles so that condition (12) holds true.

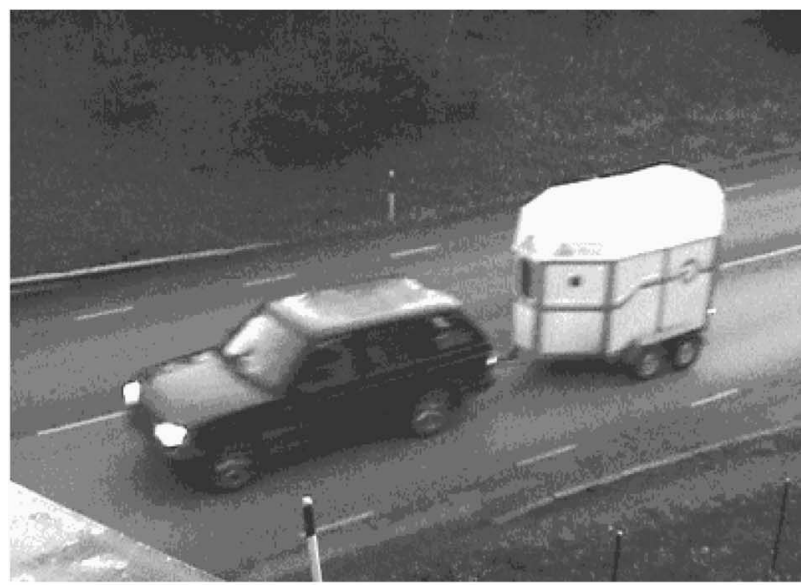

(a)

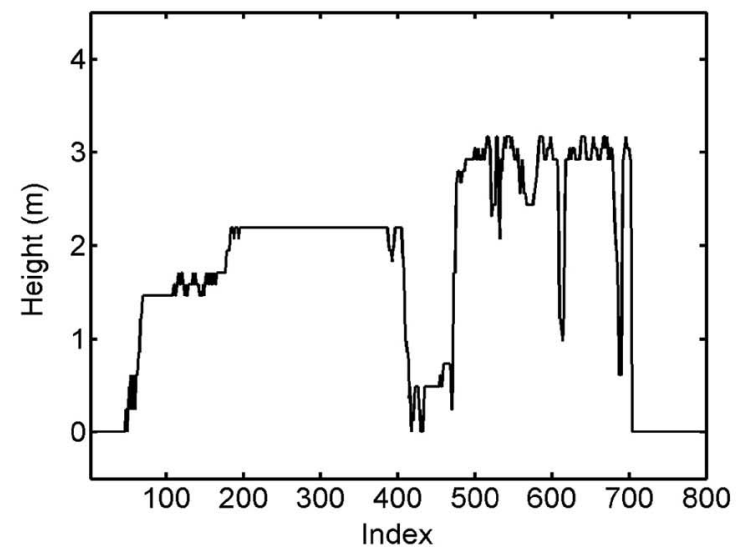

(b)

Fig. 3. (a) Image of a vehicle with trailer and (b) corresponding height samples given by the MW radar sensor.

In the second stage, the following classification algorithms were tested: 1) with making use of a full set of parameters $\{\boldsymbol{h}, h, l\}$ and 2) without making use of vehicle length $w_{i}^{L}=0$. To estimate the empirical probability of the correct classification of vehicles, two statistical tests were carried out.

In the first test, the classification scheme composed of the classes of car, car with trailer, van, bus, SUT, and MTT was used. The height and length classification boundaries used in Step 2) of the proposed classification technique are shown in Table I. For the bus classification, $n=10$ areas with center points $\left[h_{\mathrm{B} i}, l_{\mathrm{B} i}\right]^{T}, i=1, \ldots, n$ and boundaries $h_{\mathrm{B} i} \pm 0.1 \mathrm{~m}$ and $l_{\mathrm{B} i} \pm 0.5 \mathrm{~m}$ were used. The center points are shown in Fig. 1. The empirical probability of an event satisfying the decision rules specified in Steps 1) and 2) for vehicle classification was calculated for each vehicle class. The probabilities of events $P_{h l}$ are shown in Table I. As it follows from Table I, the percentage of vehicles that can be classified without making use of the height profile information is more then $80 \%$ when the vehicle length information is available and varies between $37 \%$ and $73 \%$ when the length information is not available.

For the vehicles that cannot be unambiguously classified in Steps 1) and 2), the height-profile-based feature space (2) was used in Steps 3)-5). As a metric of feature space $F^{P}$, the anisotropic Euclidian one with the diagonal weighted matrix (14) $\boldsymbol{W}=\operatorname{diag}\left\{w_{1}^{H P}, \ldots, w_{L}^{H P}, w^{H}, w^{L}\right\}$ was used, where 


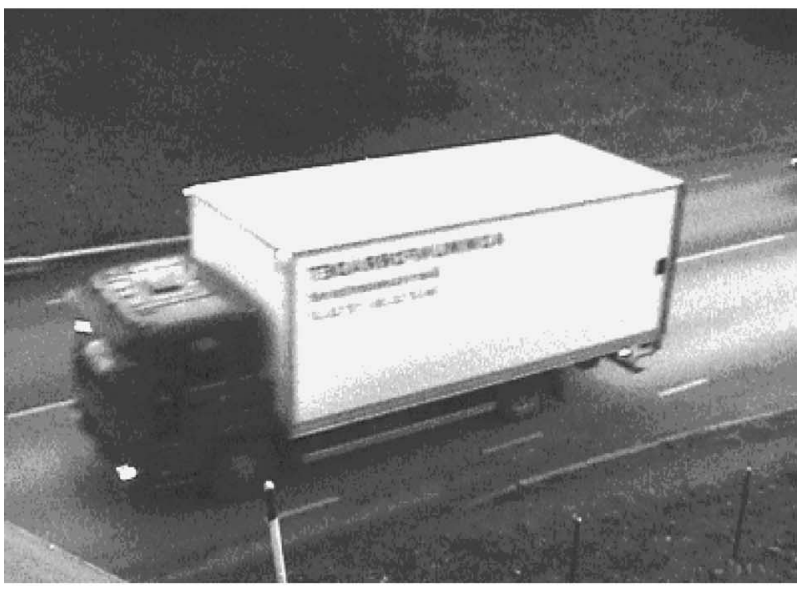

(a)

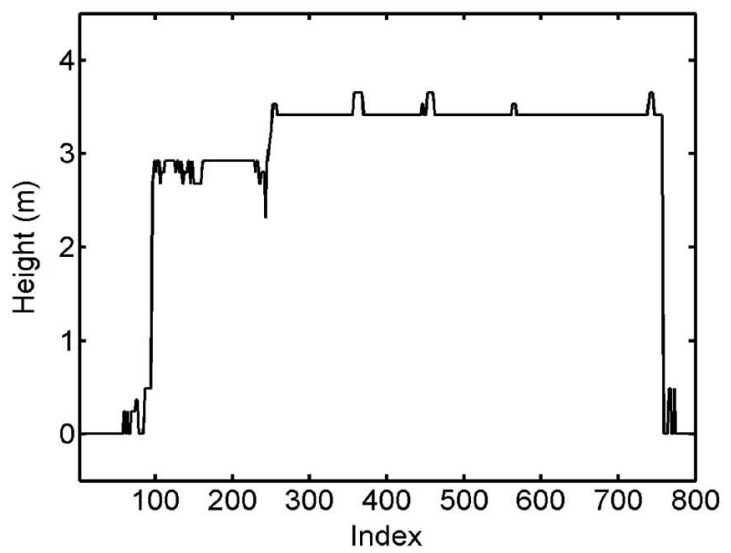

(b)

Fig. 4. (a) Image of a truck and (b) corresponding height samples given by the MW radar sensor.

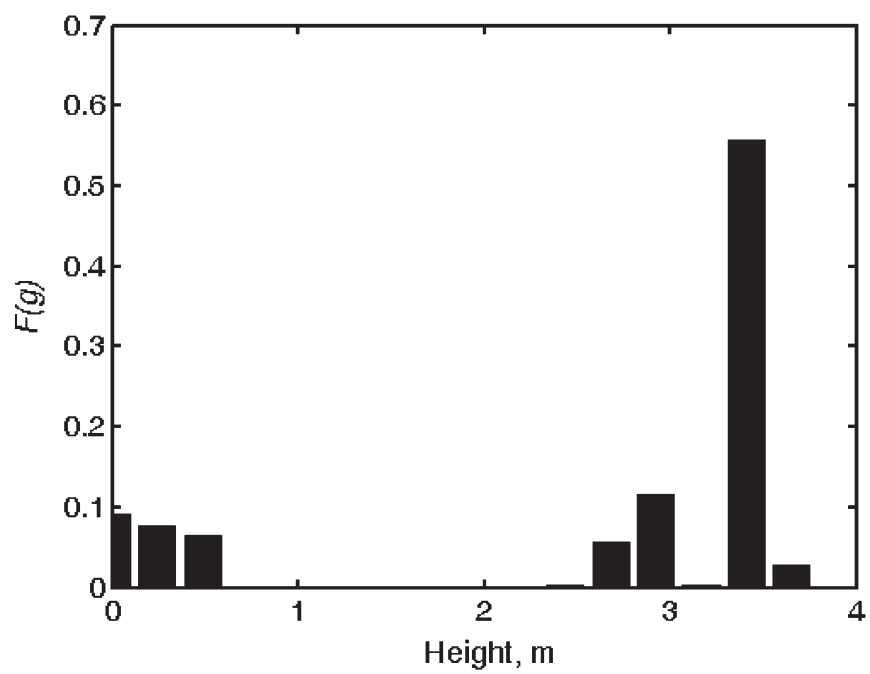

Fig. 5. Normalized histogram of the height estimates obtained for the truck shown in Fig. 4.

$w_{i}^{H P}, w^{H}$, and $w^{L}$ are the weights for height profile, height, and length, correspondingly. Taking into account the empirical RMSEs of signal parameters as well as the typical dimensions of the vehicles (see Fig. 1), the corresponding weights were chosen as $w_{i}^{H P}=1$ for all $i=1, \ldots, L$ and $w^{H}=w^{L}=5$.
TABLE I

Height AND LENGTH Classification Boundaries

\begin{tabular}{|c|c|c|c|c|c|c|}
\hline \multirow[b]{2}{*}{ Vehicle class } & \multicolumn{2}{|c|}{ Height, $m$} & \multicolumn{2}{|c|}{ Length, $m$} & \multicolumn{2}{|l|}{$P_{h l}$} \\
\hline & $\begin{array}{l}\text { Lower } \\
\text { bound }\end{array}$ & $\begin{array}{l}\text { Upper } \\
\text { bound }\end{array}$ & $\begin{array}{l}\text { Lower } \\
\text { bound }\end{array}$ & $\begin{array}{l}\text { Upper } \\
\text { bound }\end{array}$ & $w^{L}=0$ & $w^{L=5}$ \\
\hline Car & 1 & 2.1 & 2.35 & 6.5 & 0.73 & 0.85 \\
\hline Car with trailer & 1 & 2.1 & 7.4 & 15 & 0.69 & 0.89 \\
\hline Van & 2.45 & 3.17 & 4.75 & 6.5 & 0.37 & 0.75 \\
\hline Bus & 2.9 & 3.9 & 8 & 18.5 & 0.6 & 0.81 \\
\hline SUT & 3.4 & 4.6 & 6.8 & 15.5 & 0.44 & 0.85 \\
\hline ITT & 3.4 & 4.6 & 17.2 & 36.6 & 0.51 & \\
\hline
\end{tabular}

TABLE II

Field TRial Results. Test 1

\begin{tabular}{lccccc}
\hline \multirow{2}{*}{ Vehicle class } & \multirow{2}{*}{$N_{P}$} & \multirow{2}{*}{$N_{T}$} & \multirow{2}{*}{$N_{V}$} & \multicolumn{2}{c}{$P_{C C}$} \\
\cline { 5 - 6 } & & & & $w^{L=0}$ & $w^{L=5}$ \\
\hline Car & 8 & 30 & 2436 & 0.98 & 0.99 \\
Car with trailer & 5 & 12 & 128 & 0.91 & 0.98 \\
Van & 5 & 8 & 57 & 0.86 & 0.95 \\
Bus & 6 & 4 & 24 & 0.83 & 0.96 \\
SUT & 21 & 12 & 189 & 0.92 & 0.98 \\
MTT & 5 & 17 & 135 & 0.93 & 1.0 \\
Total & 50 & 83 & 2945 & 0.98 & 0.99 \\
\hline
\end{tabular}

The dimension of the vector of height profiles $L=50$. To set up pattern length $L$ and the number of patterns required $N_{p}$, a training set of 83 vehicles representing all classes was used. Both the training and tested data were verified using a ground truth camera. The number of patterns $N_{p}$, the number of vehicles to be used as the training set $N_{T}$ and to be classified $N_{V}$, and the empirical probability of correct classification $P_{C C}$ are presented in Table II.

We can see from Table II that if the estimates of both vehicle height and length are used, the probability of correct classification varies between 0.95 and 1 , depending on the classes. This result is comparable with that reported in [13]. Thus, the proposed classification technique does not decrease the classification accuracy while simplifying its practical implementation.

In the absence of vehicle length information, the probability of classification varies between 0.83 and 0.98 . Major decreases in $P_{C C}$ are found in the classification of vans and buses that have similar heights but differ in length. Thus, estimates of vehicle length can be used to enhance the classification algorithms.

In the second test, the accuracy of more detailed classification of vehicles was investigated. For this purpose, the vehicles were separated into the following subclasses: passenger car, minivan and SUV, light truck, and other. As it follows from Fig. 1, such vehicle types as minivans, SUVs, and light trucks are highly intersecting in terms of height and length. Therefore, boundaries for only two classes were used in this test. The passenger car classification was defined as $C_{1}^{*}=\{\boldsymbol{b} \mid 1.2<h \leq$ $1.5,2.4<l \leq 6.5\}$, and the "other" classification was defined as $C_{4}^{*}=\{\boldsymbol{b} \mid h>2.45, l>4.75\}$. The results of the second test show that for the passenger car classification, using Steps 1) and 2) was sufficient for the classification of $75 \%$ of vehicles when vehicle length information was available and $65 \%$ of vehicles when length information was not available. For the other classification, the corresponding percentages were $89 \%$ and $76 \%$. For a given classification scheme, the total percentage of vehicles 
TABLE III

Field Trial REsults. Test 2

\begin{tabular}{lccccc}
\hline \multirow{2}{*}{ Vehicle class } & $N_{P}$ & $N_{T}$ & $N_{V}$ & \multicolumn{2}{c}{$P_{C C}$} \\
\cline { 5 - 6 } & & & & $w^{L=0}$ & $w^{L=5}$ \\
\hline Passenger Car & 4 & 15 & 1510 & 0.78 & 0.83 \\
Minivan and & 4 & 15 & 773 & 0.75 & 0.85 \\
SUV & & & & & \\
Light Trucks & 5 & 15 & 153 & 0.81 & 0.89 \\
Other & - & - & 509 & 0.93 & 0.99 \\
Total & 13 & 45 & 2945 & 0.8 & 0.87 \\
\hline
\end{tabular}

classified using feature spaces $F^{2}=\{h, l\}$ and $F^{1}=\{h\}$ was $54 \%$ and $46 \%$, respectively. The empirical probabilities of classification are presented in Table III. They show that the proposed technique makes it possible to classify vehicle types in a more detailed level with nearly $85 \%$ accuracy. Passenger cars have approximately the same length; therefore, eliminating this parameter from the feature space does not essentially affect the probability of correct classification.

Basic physical limitations of the sensor affecting the classification performance include the antenna beam width and A/D converter capacity. Antenna beam width measured transverse to the traffic flow specifies the coverage area of the sensor. If some part of a passing vehicle is not covered by the sensor, height profile estimation accuracy may decrease. As a result, at least one sensor per lane is needed to cover the whole roadway. This limitation can be considered as a certain weakness of the down-looking sensors as compared with the side-looking ones covering multiple lines. High estimation errors can also arise when weak reflections from the vehicle surface are missed due to insufficient $\mathrm{A} / \mathrm{D}$ converter capacity. Both these limitations result in the degradation of the classification accuracy. The other causes include insufficient number of patterns and fuzzy definition of vehicle classes. Relaxing of these factors might enhance classification performance.

\section{CONClusion AND Future Work}

The problem of vehicle-type classification was addressed. A new classification technique based on using down-looking spread-spectrum MW radar was presented. The novelty of the proposed technique consists of representing different vehicle types in different feature spaces, depending on the shape and linear dimensions of the vehicles. We have shown that since many vehicle types can be unambiguously represented in terms of height and length, the necessity of using the height profiles arises only for a small portion of vehicle types for the sake of improvement of their distinguishability. As a result, the proposed classification technique involving height- and lengthbased feature space in the first stage makes it possible to avoid the need for creating many height profile-based patterns and unnecessary computations. The basic sensor requirements were formulated, and the feature extraction algorithms were presented.

The performance of the proposed approach was tested using the test-bed classification system. Field trial results have demonstrated that a detailed level of classification with high classification accuracy can be achieved in real traffic situations.
The proposed technique can be implemented using a spreadspectrum MW radar sensor that has relatively low cost, weight, and size and is largely immune to vibration, adverse weather, and illumination conditions. Further development of the system is expected to be performed in the areas of optimizing the antenna beam pattern and increasing the size and accuracy of the template library. To ensure the sufficient coverage area, the antenna beam pattern should be quite wide in the direction transverse to the traffic flow. The misclassification probability arising due to the absence of appropriate vehicle patterns can be decreased when a larger number of patterns are included to the template library. Another future development includes improvement of the signal processing algorithms such as vehicle detection and classification in a stop-and-go situation. In the stop-and-go situation, the vehicle length information is not available for the classification. Moreover, the assumption that the speed of a passing vehicle is constant does not hold. To avoid degradation of classification performance, modified classification techniques should be designed.

It is important to note that the obtained classification accuracy is close to that of the infrared laser system used at toll plazas in New York City [4]. Therefore, the proposed technique can be used for toll-collection applications. Other applications may also be suitable for bus lane enforcement, differentiated speed enforcement, statistics gathering, traffic flow control, etc.

\section{ACKNOWLEDGMENT}

The authors would like to thank J. Andersson and R. Tuoremaa for the work on the development of the early testbed system and D. Restivo for the assistance in preparing the manuscript.

\section{REFERENCES}

[1] M. Taylor and W. Young, Traffic Analysis: New Technology and New Solutions. Melbourne, Australia: Hargreen, 1988.

[2] "Vehicle classification monitoring," in Traffic Monitoring Guide, Sec. 4, U.S. Dept. Transp., Federal Highway Admin., Washington, DC, May 2001, Tech. Rep. FHWA-PL-01-021. [Online]. Available: http://www. fhwa.dot.gov/ohim/tmguide/pdf/tmg4.pdf

[3] L. E. Y. Mimbela and L. A. Klein. (2000). A Summary of Vehicle Detection and Surveillance Technologies Used in Intelligent Transportation Systems. Las Cruses, NM: The Vehicle Detector Clearinghouse. [Online]. Available: http://www.fhwa.dot.gov/ohim/tvtw/vdstits.pdf

[4] S. Tropartz, E. Horber, and K. Gruner, "Experiences and results from vehicle classification using infrared overhead laser sensors at toll plazas in New Your City," in Proc. IEEE/IEEJ/JSAI Int. Conf. Intell. Transp. Syst., Oct. 5-8, 1999, pp. 686-691.

[5] A. H. S. Lai and N. Yung, "Vehicle-type identification through automated virtual loop assignment and block-based direction-biased motion estimation," IEEE Trans. Intell. Transp. Syst., vol. 1, no. 2, pp. 86-97, Jun. 2000.

[6] T. Kato, Y. Ninomiya, and I. Masaki, "Preceding vehicle recognition based on learning from sample images," IEEE Trans. Intell. Transp. Syst., vol. 3, no. 4, pp. 252-259, Dec. 2002.

[7] J.-W. Hsieh, S.-H. Yu, Y.-S. Chen, and W.-F. Hu, "Automatic traffic surveillance system for vehicle tracking and classification," IEEE Trans. Intell. Transp. Syst., vol. 7, no. 2, pp. 175-177, Jun. 2006.

[8] H. Roe and G. S. Hobson, "Improved discrimination of microwave vehicle profiles," in Proc. IEEE MTT-S Int. Microw. Symp., Jun. 1-5, 1992, pp. 717-720.

[9] E. K. Walton, I. P. Theron, S. Gunawan, and L. Cai, "Moving vehicle range profiles measured using a noise radar," in Proc. IEEE Antennas and Propag. Soc. Int. Symp., 1997, vol. 4, pp. 2597-2600. 
[10] M. Cherniakov, R. S. A. R. Abdullah, P. Jancovic, and M. Salous, "Forward scattering micro sensor for vehicle classification," in Proc. RADAR Conf., 2005, pp. 184-189.

[11] S. J. Park, T. Y. Kim, S. M. Kang, and K. H. Koo, "A novel signal processing technique for vehicle detection radar," in Proc. IEEE MTT-S Int. Microw. Symp., Jun. 8-13, 2003, vol. 1, pp. 607-610.

[12] M. Jahangir, K. M. Ponting, and J. W. O'Loghlen, "Robust doppler classification technique based on hidden markov models," Proc. Inst. Electr. Eng.-_Radar Sonar Navig., vol. 150, no. 1, pp. 33-36, Feb. 2003.

[13] I. Urazghildiiev, R. Ragnarsson, K. Wallin, A. Rydberg, P. Ridderström, and E. Öjefors, "A vehicle classification system based on microwave radar measurement of height profiles," in Proc. RADAR Conf., 2002, pp. 409-413.

[14] M6 Toll, General Information. Vehicle Classification. [Online]. Available: http://www.m6toll.co.uk/

[15] R. Duda, P. Hart, and D. Stork, Pattern Classification. Hoboken, NJ: Wiley, 2001

[16] A. Webb, Statistical Pattern Recognition. Hoboken, NJ: Wiley, 2002.

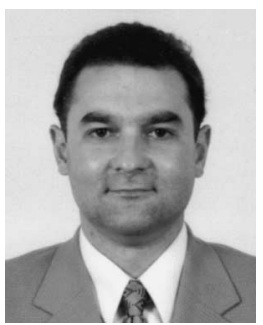

Ildar Urazghildiiev received the Ph.D. degree in electrical engineering from the National R\&D Center of Defense Technologies and Military Security of Ukraine, Kiev, Ukraine, in 1996.

From 1994 to 2001, he was with the National R\&D Center of Defense Technologies and Military Security of Ukraine and the National Technical University of Ukraine "Kiev Polytechnic Institute," Kiev. In 2001 and 2003, he held a Postdoctoral Fellow position at the Signals and Systems Group, Uppsala University, Uppsala, Sweden. In 2004, he joined the Laboratory of Ornithology, Cornell University, Ithaca, NY, where he is currently a Mathematician-Programmer. His research interests include signal processing and signal detection, parameter estimation, classification, localization, and tracking with applications to radar and bioacoustics.

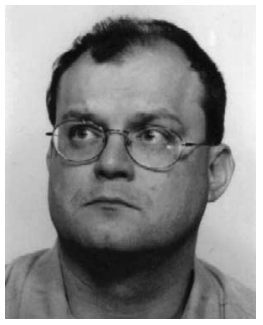

Rolf Ragnarsson received the M.Sc degree in engineering from Uppsala University, Uppsala, Sweden, and the M.Sc degree in physics from Cornell University, Ithaca, NY.

$\mathrm{He}$ is currently a Development Engineer with Sensys Traffic AB, Uppsala, where he leads the development of a classification radar system.

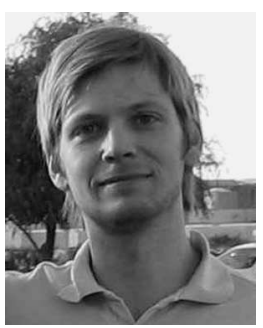

Pierre Ridderström received the M.Sc. degree from Uppsala University, Uppsala, Sweden.

He is currently with Sensys Traffic AB, Uppsala, as an RF Designer.

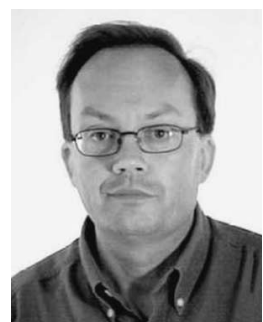

Anders Rydberg (M'89) received the M.Sc. degree from Lund Institute of Technology, Lund, Sweden, in 1976 and the Ph.D. degree from Chalmers University of Technology, Gothenburg, Sweden, in 1988.

From 1977 to 1983 , he was with the National Defence Research Establishment, ELLEMTEL Development Company, and the Onsala Space Observatory. In 1991, he was appointed Docent (Associated Professor) at Chalmers University of Technology. From 1990 to 1991, he was a Senior Research Engineer with Farran Technology Ltd., Cork, Ireland. He is currently with the Department of Signals and Systems, Uppsala University, Uppsala, Sweden, where he was an Associated Professor in 1992 and has been a Professor in applied microwave and millimeter-wave technology since 2001. He has authored and coauthored more than 140 publications in the area of micro- and millimeter-wave antennas and solid-state components and circuits. He holds three patents in these areas.

Prof. Rydberg is a member of the Editorial Board of the IEEE TRANSACTIONS ON MiCROWAVE THEORY AND TECHNiQues, an adjunct member of Sections B and D of the Swedish Member Committee of URSI (SNRV), and the Chairman for the Swedish IEEE MTT/AP Chapter.

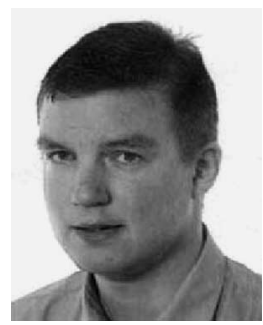

Erik Öjefors received the M.Sc. degree in engineering physics from Uppsala University, Uppsala, Sweden, in 2000 . He is currently working toward the $\mathrm{Ph} . \mathrm{D}$. degree in microwave technology at the Department of Signals and Systems, Uppsala University.

His research interests include monolithic integration of SiGe circuits with micromachined passive components and system-on-a-chip implementation of millimeter-wave transceivers.

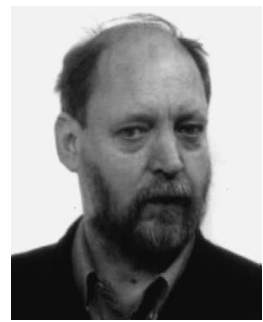

Kjell Wallin received the M.S. degrees in electrical engineering from the Royal Institute of Technology, Stockholm, Sweden, in 1976.

He has been managing various research and development projects in the telecommunication area for Ericsson Radio Systems and in the traffic application area for Sensys Traffic AB, Jönköping, Sweden, since 1977.

Per Enochsson, photograph and biography not available at the time of publication.

Magnus Ericson, photograph and biography not available at the time of publication.

Göran Löfqvist, photograph and biography not available at the time of publication. 